\title{
An Evaluation of Opportunistic Native Multicast
}

\author{
Dhaifallah Alwadani, Mario Kolberg \\ Computing Science and Mathematics \\ University of Stirling \\ Stirling, Scotland \\ \{dba,mko\}@cs.stir.ac.uk
}

\author{
John Buford \\ Avaya Labs Research \\ Basking Ridge, New Jersey, USA
}

\begin{abstract}
Hybrid multicast opportunistically combines Application Layer Multicast and native multicast protocols. This paper presents an evaluation of Opportunistic Native Multicast which uses the AMT multicast tunnelling protocol.

We describe our opportunistic multicasting approach that tries to solve the islands phenomenon by building unicast tunnels to connect these islands while attempting to utilise the native multicast capability of the islands.

It is expected that this hybrid approach will improve both efficiency and availability of multicast. We compare our approach to the ALM protocol SCRIBE.

The comparison here was done using five metrics: Stress, Stretch, intra-island traffic, interisland traffic and Delivery rate. In all of them, our proposed model has shown improved results over ALM. Moreover, we investigated what effect the number of islands that the receivers are distributed into, has on performance.
\end{abstract}

\section{INTRODUCTION}

Different Internet application, such as IPTV and conference calls, rely on distributing content in a one-to-many or many-to-many approach. This way of content delivery is called multicasting. Multicasting is very powerful and efficient way to deliver content in the Internet. It was designed to save bandwidth and manage the routing and delivering of content to multiple destinations such as media delivery and update pushing. This made multicasting critical since $91 \%$ of the Internet traffic is expected to be video according to the Cisco Visual Networking Index [1].

To achieve multicasting, there exist two techniques. The first technique is to rely on routers to forward and replicate the multicast message. In this case, routers form a spanning tree for each multicast group. This technique is called Native Multicast. Another technique is Application Layer Multicast ALM. Here, hosts, not routers, are responsible on forming the spanning tree. Also, hosts will do the replicating and forwarding of the multicast messages.

ALM does not require multicast support from the routers but it is not as efficient as Native Multicast. However, to be able to use native multicasting, the routers need to be multicast-capable. Currently, this is not always the case across the entire Internet [2]. This has led to multicast- capable parts of the Internet forming islands which are not connected by multicast capable links. This scenario is depicted in Figure 1. While the use of multicast-capable routers is increasing, the problem of multicast islands still exists. Until global adoption of native multicasting is achieved, content providers will have to rely on unicast to distribute their content.

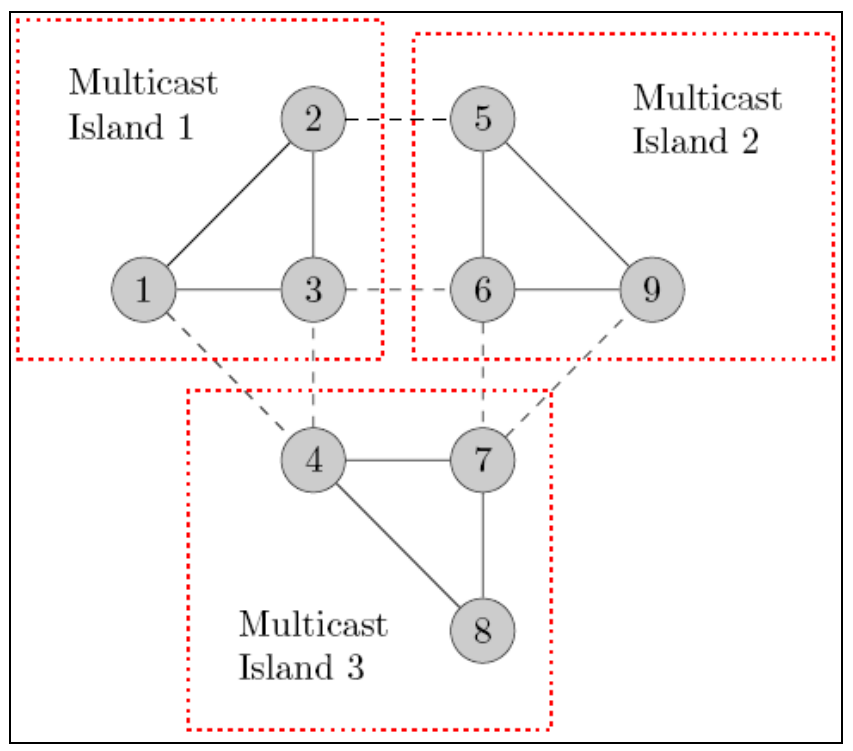

Figure 1: Example of Multicast Islands.

However, a solution to this problem is to connect these multicast islands using Application Layer Multicast. By doing so, the benefits of both techniques can be gained. This approach is called Hybrid Multicasting. While Hybrid Multicast can provide a great improvement in efficiency over ALM it has not been given sufficient attention [3]. In this paper, the main focus will be on designing and analysing a framework for Hybrid Multicasting using peerto-peer overlays.

\section{RELATED WORK}

One of the earliest attempts on connecting multiple native multicast islands was MBone which was introduced in the early 1990s [4]. Based on this approach, other research was carried out to connect islands to the MBone. However, these approaches required the installation of dedicated 
hardware and software. Additionally, an administrator must build and maintain these tunnels. These reasons have rendered these solutions impractical as a universal approach as they are neither scalable nor dynamic.

Automatic Multicast Tunnelling (AMT) was proposed to automate the management of tunnels [5]. AMT provides specific devices on the networks, AMT Relays and AMT Gateways, to interact with the underlying native multicast protocol and to build tunnels when needed. However, the AMT approach focuses on connecting island pairs rather than building a global unified network. Moreover, the required changes to the network infrastructure of the islands required to add support in the routers, or alternatively, required the installation of additional devices. Furthermore, AMT does not deal with changes to the network topology. One other drawback of AMT is the lack of some features such as resource locating and management. A solution, which extends RELOAD [17] to work with AMT is presented in [15] and [16].

Subset Multicast (SM) tries to solve the issue of connecting multicast islands by having the source island forward a copy of the data to each island [6]. While SM works independently from the underlying native multicast protocol, a source must have a database of every interested island and manage the database. Like with other techniques, this causes scalability issues with SM.

As has been proposed in [7], Universal Multicast (UM) provides a way to connecting multicast islands using dynamically built unicast tunnels. UM allows for multiple connections linking pairs of islands. Doing so will allow for an increased throughput with large islands. Inside each multicast island, one or more Dedicated Members (DM) are elected to natively deliver the multicast to the island's nodes. Also, the authors of Universal Multicast have proposed a protocol for intra-island multiple-DM management protocol [8]. This protocol is called Host Group Management Protocol HGMP. HGMP is concerned with electing peers to be DM in an island dynamically and using multiple DMs together for load-balancing purposes.

In [3], the authors utilise overlay protocols to build and manage the unicast tunnels. They describe the two different protocols that are to be used to connect the islands:

Centralized Island Multicast CIM: This protocol is suitable for small sized groups that have many-to-many communication and high bandwidth requirement e.g. multi-party conference calls. Here, there is a central server to build and maintain the spanning tree.

Distributed Island Multicast DIM: This protocol is suitable for large groups where scalability is required. In DIM, hosts in the same multicast island elect a unique leader. The leader node will be responsible the delivery overlay.

\section{OpPORTUNISTIC NATIVE MUlticAST}

The proposed model takes advantage of cross layer awareness combining information from different layers to make decisions. Decisions are needed for the discovery of islands and the mechanism to elect nodes. By accessing the information available in the lower layers (Network and Data Link), ALM peers are aware of the status of the underlay including the support of native multicast and the existence of other peers in the same multicast domain. Peers joining the same ALM group who are also located in the same native multicast island communicate using native multicast for intra-island communication.

Consequently, the proposed model will join application layer trees with native multicast trees. At the application layer, it makes use of P2P overlays opportunistically, combining ALM trees and native multicast where available and linking multicast islands using AMT.

In the approach, peers optimize the way that they are connected to the multicast tree. Hosts inside an island will elect which nodes will directly connect to the ALM tree. This elected peer will be responsible for relaying the information between the ALM and native multicast trees. Thus, the approach will distinguish the following three types of communication: Intra-Island, Inter-Island and Overlay Communications.

The multicast tree uses $\mathrm{P} 2 \mathrm{P}$ overlay connections to elected nodes in each island. Within an island, peers use native multicast to relay traffic. Between islands, elected peers use the AMT protocol to tunnel traffic. Figure 2 shows an example of 6 multicast islands. These Islands are connected using R1 and R2 which do not support Native Multicast. Elected Nodes, represented here by N1-6, form a tree of AMT tunnels.

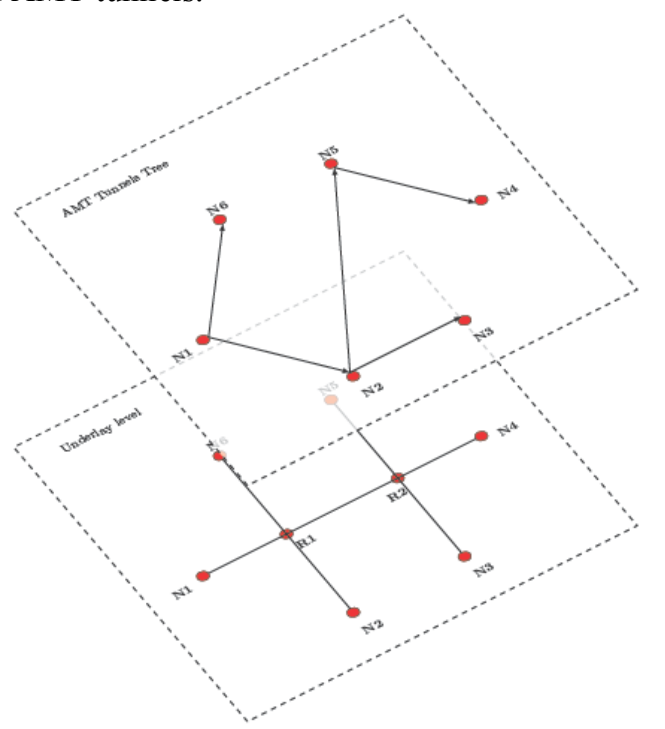

Figure 2: Example of Multicast Islands.

The proposed hybrid approach improves both, efficiency and availability of multicast communication as well as its scalability and adaptability. 


\section{A. Island Discovery and Election}

Each island should choose a node that is part of the ALM tree and can use the native multicast capability of the network. So, each node will send an election message to the network to the multicast address corresponding to the ALM group ID. Every node that is interested in being a relaying node must participate in this communication. Currently, the node with the lowest IP address will be the relay. This may not prove to be the optimum choice but this can be refined in the future. After the node becomes the relaying node, it keeps sending the election message every 10 seconds. Again, this interval will be subject to a further more detailed study in the future. The re-election will happen whenever the network misses 3 such messages from the relaying node.

\section{B. AMT Tunnels}

Automatic Multicast Tunneling (AMT) is used to allows for connectivity between multicast islands without the need of explicit tunnels. Also, it allows users who are connected to a unicast-only network to join in multicast groups. Using the AMT tunnel, the traffic will be encapsulated in UDP packets, which will be sent as a unicast message through the unicast only network.

AMT uses the client-server approach. Without the use of AMT, if the host connected to the unicast-only network were to try to send IGMP messages, the network would drop these packets due to the fact that the network does not support multicast. Alternatively, a process in these hosts may directly intercept such requests. In order to setup an AMT tunnel, an AMT request will be sent towards the AMT relay.

This will establish a tunnel between the gateway and the relay using a 3-way handshake. With the tunnel in place, any IGMP membership update messages will be encapsulated in the AMT tunnel. The AMT relay will decapsulate the IGMP membership report and will trigger PIM join towards the source. Finally, the AMT relay will send any multicast traffic to the hosts that are interested by encapsulating them in the tunnel.

ONM uses AMT tunnels to encapsulate traffic between islands. The use of AMT tunnels is an essential part of ONM as it can provide the following advantages to ONM:

- $\quad$ AMT provides native support for multicasting. Nodes in the islands can use native multicast protocols, e.g. IGMP, to participate in multicast groups.

- Keeping the Layer-4 information in the packets allowing for better QoS and traffic shaping.

- Standardisation. This will be important when dealing with connecting islands under different management.

- Islands that do not wish to participate in the overlay can use AMT devices to connect to an island that is part of the overly.

- Utilising the security mechanism already available in AMT.

\section{EXPERIMENTATION}

\section{A. Framework}

Evaluating hybrid multicast protocols is difficult for two reasons. The simulation environment must combine both a scalable overlay and a detailed network layer that includes routers with NM support. The authors have previously studied a related problem with parallelizing overlay messaging with multi-destination multicast routing [12]. There are a number of network simulators but we have chosen OMNeT++ [13][14] with the INET model together with Oversim [9] for a number of reasons including:

Extensible and Modular: This is a must have feature since there currently is no implementation for AMT in any existing simulator. Omnet++ is open source and hence freely extendible. One of the most important features of $\mathrm{OMNeT}++$ is its modular design. OMNeT++ supports simple modules and compound modules. The latter groups other modules together and describes how these sub-modules are connected. Compound modules are written using the NED language, which is a topology description language. On the other hand, simple models are written in $\mathrm{C}++$ and implement protocol logic. Omnet's hierarchal structure helps reusing some of the existing code in new protocol models and makes it possible to share modules in the open source community.

INET model: This model provides a wide range of models of several Internet protocols which include IGMP.

Oversim: is built on top of Omnet++ and provides a framework to simulate peer-to-peer overlay protocols. It includes models of many common protocols such as Pastry and its ALM algorithm Scribe. It can achieve large network sizes with 10s of thousands of nodes and is able to interface with INET to include specific underlay behaviour in the simulation.

Suitable evaluation metrics are needed to compare the tree quality of our approach with pure overlay multicast trees. Due to the lack of a suitable simulator that integrates the required tools to test our proposed model, the authors have previously presented an approach to simulating and evaluating hybrid multicast protocols using the Omnet++/INET/Oversim engine and protocol stack [10].

\section{B. Metrics}

To fully understand and compare our new approach, we employ the following metrics, which will serve as a base of our comparison:

Stretch: is the delay of the overlay path over the delay of a unicast message [11].

Stress: is the number of identical copies of a message carried by a link or a node [11].

Delivery Rate: is the percentage of the message received over the number of message that the node should receive.

Intra-island Traffic: The traffic that is routed inside the island.

Inter-island Traffic: the amount of traffic crossing the backbone. 
We expect our system to achieve better results with these metrics i.e. Stress and Stretch. This is due to the considerably lower number of nodes participating in the ALM tree. Moreover, since traffic utilizes native multicast where available, the stress on the backbone is minimized to the theoretical limit of ALM.

\section{Simulation Model}

In order to test the proposed hybrid multicast framework and our implementation in Omnet++/Oversim, we set up a number of experiments. Our experiments use a network that contains a multicast group of 2000 nodes. This group is distributed across different numbers of islands ranging from 10 up to 150 islands. In the experiments a source node sends multicast traffic at a rate of 1 packet every 5 seconds. We will compare our approach with pure ALM and pure NM. In the experiments we use Scribe as the ALM protocol. This leads to the following three configurations:

ALM: Scribe is used to manage the group and distribute the multicast data. There is no native multicast support anywhere in the network.

Native Multicast: IGMPv2 is used to manage the groups and distribute multicast data. All the island and the backbone routers support IGMP.

ONM: The islands support IGMP, but the backbone is unicast only. This essentially models native multicast islands. We have 200 ONM devices distributed randomly in the network. These devices will form an ALM tree and discover the topology capability. Furthermore, these devices are responsible for distributing the data across the remainder of the 2000 devices.

Due to the many ways that a tree in ALM could form, it was very susceptible to the seed value of the simulator. To mitigate the effect, we repeated each scenario 15 times with different seed values for the simulator's random number generator. By doing so, a clear pattern emerged.

\section{RESUlts}

The stretch of the multicast message depends heavily on the number of nodes participating on the overlay. In the case of the pure ALM, the is no noticeable difference with the nodes being divided into different numbers of islands. So, ALM should not be affected by the number of islands. However, in the case of ONM, the size of the ALM tree, and subsequently the stretch, will depend on how many islands there are in the topology. Thus the stretch should increase with an increased number of islands.

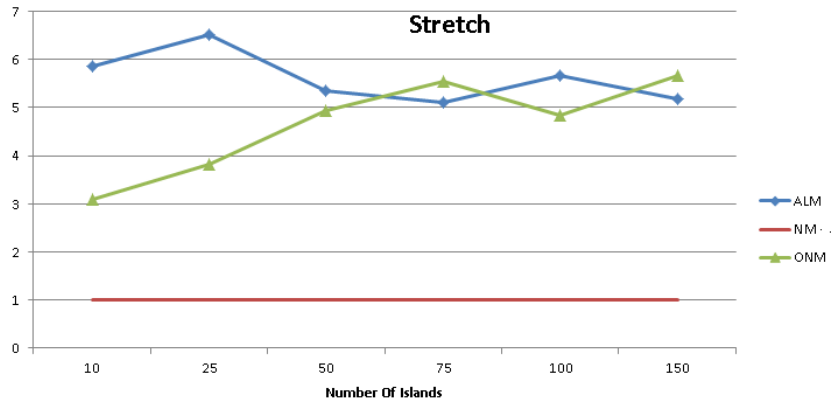

Figure 3: Comparing the Stretch of different multicast approaches.

In Figure 3, with a small number of islands, we can see that ONM results in a better stretch value in the overlay when compared with ALM. We can also see that the stretch is not linked to the number of islands with pure ALM. After a certain threshold, the stretch of ONM would converge with that of ALM as the number of islands is too large to give ONM any benefit in terms of stretch.

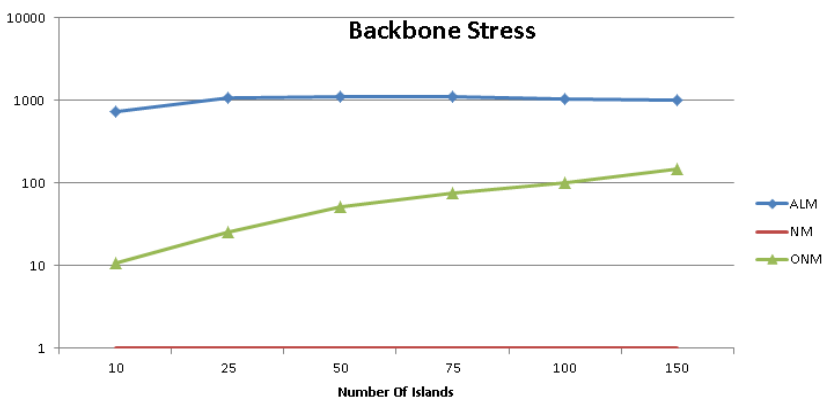

Figure 4: Comparing the Stress of different multicast approaches.

From Figure 4, we can see that the ALM Stress on the backbone is exponentially greater than ONM's. This is due to fewer copies of a messages being sent between islands. In the case of ALM we have a linear correlation between the number of receivers and the stress on the backbone. While ONM results in significantly lower stress on the backbone than for ALM, the stress with ONM increases with the number of islands.

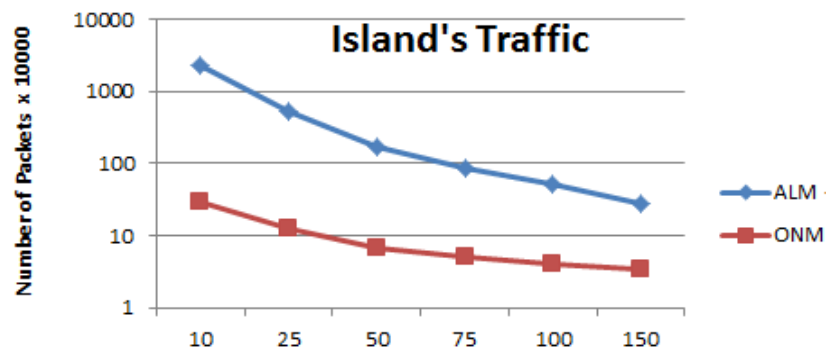

Figure 5: Comparing the traffic generated in each island for different multicast approaches 
Figure 5 displays the number of packet routed inside the islands. With a fixed number of nodes such as in our case, as the number of islands increases, the average number of nodes per island decreases. This would result in less traffic inside the islands. Crucially, we can see that ONM produce significantly less traffic inside the island when compared with ALM.

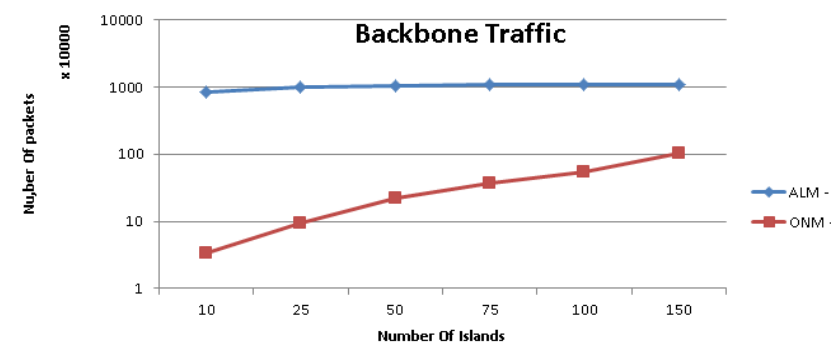

Figure 6: Comparing the traffic crossing the backbone for different multicast approach.

In Figure 6, the traffic crossing the backbone does not depend on the number of islands with ALM. As expected, in the case of ONM the traffic on the backbone increases with the increase in the number of islands.

\section{CONCLUSIONS}

We have presented our approach of delivering Multicast in an environment that does not offer universal support for Native Multicast. Our approach creates an ALM tree and connects hosts that are unable to connect to the tree source natively. To optimise the performance of the delivery, the nodes that are present in the same multicast-enabled island will elect one of the nodes to act as an AMT device allowing other nodes to utilise native multicast to propagate the information inside the island. Clearly, this will decrease the number of copies of the same message to cross the backbone.

For the purpose of evaluation, we have identified five metrics: Stress, Stretch, intra-island traffic, inter-island traffic and delivery rate. Our approach has shown better results than pure ALM in every metric. However, as can be expected, the performance decreases with the increase of the number of islands. In the extreme case, if the number of islands becomes too large, ONM will yield similar results as ALM (every node would form its own single-node island).

\section{REFERENCES}

[1] CISCO. Cisco Visual Networking Index: Forecast and Methodology, 2012 - 2017, White Paper, pages 1-10, 2013.

[2] C. Diot, B. Levine, and B. Lyles, "Deployment issues for the IP multicast service and architecture," IEEE Network, Vol. 14(1), pp. 78-88, 2000. http://ieeexplore.iee. $\mathrm{org} / \mathrm{xpls} / \mathrm{abs}$ all.jsp?arnumber $=819174$

[3] X. Jin, KL Cheng, and SHG Chan. Island multicast: combining IP multicast with overlay data distribution. IEEE Transactions Multimedia, 11(5): 2009 $\underline{\mathrm{http}: / / \text { ieeexplore.iee. } \mathrm{org} / \mathrm{xpls} / \mathrm{abs} \text { all.jsp?arnumber }=490711}$ $\underline{3}$
[4] H. ERIKSSON, "MBone: the multicast backbone," Communications of the ACM, vol. 37, pp. 54-60, 1994.

[5] G. Bumgardner. Automatic Multicast Tunneling (AMT). IETF RFC7450 https://tools.ietf.org/html/rfc7450 Feb. 2015.

[6] Juyoung Park, Dae Young Kim, Shin Gak Kang, and Seok Joo Koh. Multicast delivery based on unicast and subnet multicast. IEEE Communications Letters, 5(4):181-183, April 2001. http://ieeexplore.ieee.org/lpdocs/epic03/wrapper.htm?arnumb er $=917107$

[7] Sugih Jamin, Lixia Zhang, and Beichuan Zhang. Host multicast: a framework for delivering multicast to end users. 21st Joint Conference of the IEEE Computer and Communications Societies, 3:1366-1375, 2002. http://ieeexplore.ieee.org/lpdocs/epic03/wrapper.htm?arnumb $\underline{\mathrm{er}}=1019387$

[8] Beichuan Zhang, Sugih Jamin, and L Zhang. Universal IP multicast delivery. Int'l Workshop on Networked Group Communication, 2002.

[9] I. Baumgart, B. Heep, S. Krause. OverSim: A Flexible Overlay Network Simulation Framework, IEEE Global Internet Symposium 2007.

http://ieeexplore.ieee.org/lpdocs/epic03/wrapper.htm?arnumb er $=4301435$

[10] D. Alwadani, M. Kolberg, and J. Buford, "A Simulation Model for Hybrid Multicast," 2014 Eighth International Conference on Next Generation Mobile Apps, Services and Technologies, pp. 112-116, 2014.

http://ieeexplore.ieee.org/lpdocs/epic03/wrapper.htm?arnumb er $=6982901$

[11] Y.-H. Chu, S. G. Rao, and H. Zhang. A Case for End System Multicast. In Proceedings of ACM SIGMETRICS, June 2000.

[12] J. Buford, A. Brown, M. Kolberg. Exploiting Parallelism in the Design of Peer-to-Peer Overlays. J. Computer Communications. Special Issue on Foundations of Peer-toPeer Computing Vol 31/3, Feb. 2008, pp 452-463.

[13] Andreas Varga and Rudolf Hornig. An overview of the $\mathrm{OMNeT}++$ simulation environment. 1st Intl. Conf. on Simulation, 2008.

[14] A. Varga. Omnet++ community site. http://www.omnetpp.org

[15] M. Kolberg (Ed.), J. Buford. Application-Layer Multicast Extensions to REsource LOcation And Discovery (RELOAD), RFC 7019, IRTF, 2013. https://tools.ietf.org/html/rfc7019

[16] M. Kolberg and J. Buford. Application Layer Multicast extensions to RELOAD. 2011 IEEE Consumer Communications and Networking Conference (CCNC), pages 1083-1087, January 2011.

[17] C. Jennings, B. Lowekamp, E. Rescorla, S. Baset and H. Schulzrinne. REsource LOcation And Discovery (RELOAD) Base Protocol, RFC 6940, IETF, 2014. https://tools.ietf.org/html/rfc6940 